\title{
EMITTANCE MEASUREMENT FROM THE DECAY RATE OF COHERENT OSCILLATION THROUGH NONLINEAR FILAMENTATION
}

\author{
S. Kamada, N. Akasaka and K. Ohmi \\ KEK, 1-1 Oho, Tsukuba-shi, Ibaraki-ken, 305 Japan
}

\begin{abstract}
A completely new approach to measure beam emittance is examined. It is based on measuring the decay rate of coherent betatron-oscillation through nonlinear filamentation caused by lattice nonlinearity. Experimental results, computer simulations and analytic approach using differential algebra (DA) [1] [2] are presented. This method may provide useful tools in measuring emittance at high brightness light sources, high luminosity particle factories and damping rings for linear colliders where emittance and coupling are expected to be very small.
\end{abstract}

\section{INTRODUCTION}

Emittance measurement is an essential issue for tuning performance of accelerators, but it can not be done easily in case of very low emittance.

In these days, it has become an established technique that coherent oscillation of beam excited by a kicker magnet is detected using a single passage beam position monitor and recorded turn by turn for studies of beam dynamics.[3]

A beam consisting of many particles with slightly different betatron tune shows decay of coherent oscillation through nonlinear filamentation, in which the motion of beam mass center damps while oscillation amplitude of each particle remains constant.[3][4]

Amplitude dependence of the decay rate through nonlinear filamentation has been observed experimentally at the TRISTAN main ring as shown in Fig. 1. In section 2, the decay rate will be derived analytically from Hamiltonian whose nonlinear coefficients should be calculated by DA. The decay rate depends on initial beam emittance before kick and lattice nonlinearity as well as amplitude of the kick.

To simulate emittance measurement from the decay rate, multi-particle tracking by the SAD code [7] was conducted using interleaved and non-interleaved optics of the TRISTAN main ring and optic for the low energy ring of KEK B-factory (KEKB-LER). Each of these optics has unique character of lattice nonlinearity. It will be introduced in section 3.

Results of the simulation and the analytic treatment will be presented in section 4 . They will be compared and discussed.

\section{THEORY}

\subsection{Hamiltonian and tune}

Applying the canonical perturbation, one turn Hamiltonian for a particle in a circular accelerator is given as (1) in

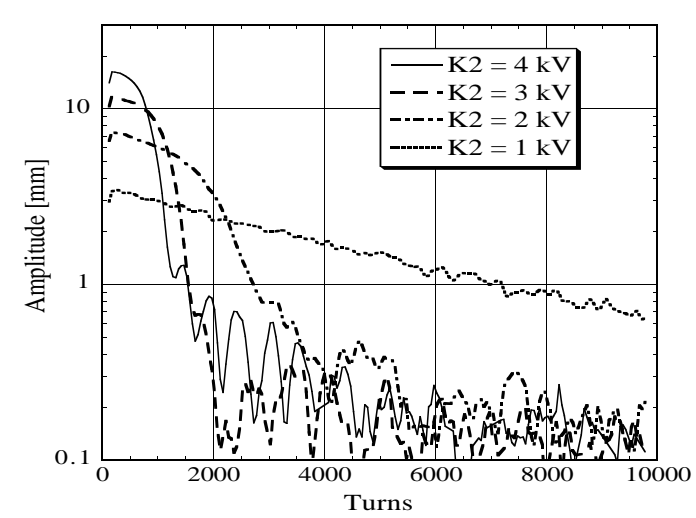

Figure 1: Decay of vertical oscillation through nonlinear filamentation. The data were taken at various kicker voltages $K 2$ in non-interleaved sextupoles optic of the TRISTAN main ring. The decay curve is characterized by parabola and the decay rate grows at the larger amplitude oscillation. The beam current was kept around $30 \mu \mathrm{A}$ to reduce damping by head-tail effect.

action-angle form up to the 2-nd order.

$\mathcal{H}=\sum_{w \in(x, y, z)}\left\{2 \pi \nu_{w}^{0} J_{w}+a_{w w} J_{w}^{2}\right\}+\frac{1}{2} \sum_{u, v \in(x, y, z)}^{u \neq v} a_{u v} J_{u} J_{v}$

where $x, y$ and $z$ represent horizontal, vertical and longitudinal coordinates, respectively and $a_{u v}$ describes the lowest order nonlinearity of the system.

Tune of a particle in $w$-coordinate is obtained by differentiating the Hamiltonian by action $J_{w}$ partially,

$$
\nu_{w} \equiv \frac{1}{2 \pi} \frac{\partial \psi_{w}}{\partial n}=\frac{1}{2 \pi} \frac{\partial \mathcal{H}}{\partial J_{w}}
$$

A beam, a set of many particles of different actions, looses coherence of oscillation phase because of the tune-spread caused by (2), that is called nonlinear filamentation.

\subsection{Distribution of action}

Before a coherent kick or displacement, particles' distribution in a beam is assumed to be Gaussian in phase space of coordinate-momentum form and described by the distribution function of action as

$$
f_{w}\left(J_{w}\right)=\frac{1}{\varepsilon_{w}} e^{-J_{w} / \varepsilon_{w}},
$$

where $\varepsilon_{w}$ represents beam emittance in $w$-coordinate. 
By coherent vertical displacement of the beam, the distribution function of $J_{y}$ becomes,

$$
f_{y}\left(J_{y}\right)=\frac{1}{\varepsilon_{y}} e^{-\left\{J_{y}+\frac{1}{2}\left(\frac{\Delta_{y}}{\sqrt{\beta_{y}}}\right)^{2}\right\} / \varepsilon_{y}} I_{0}\left(\frac{\Delta_{y}}{\sqrt{\beta_{y}}} \frac{\sqrt{2 J_{y}}}{\varepsilon_{y}}\right),
$$

where $\Delta_{y}, \beta_{y}$ and $I_{0}$ stand for amount of displacement, $\beta$ function at the displacement place and the modified Bessel function of 0 -th order, respectively.

\subsection{Distribution of tune}

Standard deviation of tune in $y$-coordinate just after the coherent displacement is obtained by taking the following ensemble mean using the distribution functions given by (3) and (4).

$$
\sigma_{\nu_{y}}=\sqrt{\left\langle\nu_{y}^{2}\right\rangle-\left\langle\nu_{y}\right\rangle^{2}}
$$

This gives,

$$
\sigma_{\nu_{y}}=\frac{1}{2 \pi} \sqrt{a_{x y}^{2} \varepsilon_{x}^{2}+a_{y z}^{2} \varepsilon_{z}^{2}+4 a_{y y}^{2} \varepsilon_{y}^{2}\left\{1+\left(\frac{\Delta_{y}}{\sqrt{\varepsilon_{y} \beta_{y}}}\right)^{2}\right\}} .
$$

When coherent displacement is much larger than initial emittances, (6) the following approximation is valid.

$$
\sigma_{\nu_{y}}=\frac{1}{\pi}\left|a_{y y}\right| \sqrt{\frac{\varepsilon_{y}}{\beta_{y}}} \Delta_{y}
$$

\subsection{Decay rate of coherent oscillation}

Assuming that particles in a beam have same radial amplitude $\sqrt{2 J_{y}(0)}$ and azimuthally allocate in Gaussian distribution of standard deviation $\sigma_{\theta}$. Then the radial amplitude of mass center motion becomes smaller than $\sqrt{2 J_{y}(0)}$ by factor $e^{-\sigma_{\theta}^{2} / 2}$.

$n$-turns after coherent displacement of a beam, tunespread of the beam given by (6) causes azimuthal distribution of particles and reduces the radial amplitude of mass center motion as in the following.

$$
\sqrt{\frac{J_{y}(n)}{J_{y}(0)}}=e^{-\sigma_{\theta}^{2} / 2}=e^{-\left(2 \pi \sigma_{\nu y} n\right)^{2} / 2} \equiv e^{-\left(n / n_{N F}\right)^{2}}
$$

where $J_{y}(n)$ stands for action of mass center motion $n$ turns after the coherent displacement. Eq. (8) also defines $n_{N F}$, damping time of coherent oscillation through nonlinear filamentation. In experimental measurements, $n_{N F}$ is observable and gives tune-spread of the beam.

$$
\sigma_{\nu_{y}}=\frac{1}{\sqrt{2} \pi n_{N F}}
$$

\section{TRACKING SIMULATION}

\subsection{Method of simulation}

Multi-particle tracking was conducted by SAD code, typically with 300 particles for 1000 turns. Radiation damping and quantum fluctuation were included.

Tracking result, a series of mass center position, was treated in the same way made in the experiments.[4] The instantaneous spectrum is calculated with a series of data which corresponds to $N_{b i n}$ turns. The Hanning window is adopted as a weight function. In order to find the frequency and amplitude of the peak precisely, the true continuous spectrum around the peak is approximated by a parabola fitted to the largest three points. The time variation of the spectrum is obtained by shifting the data by $N_{\text {step }}$ turns. We adopted $N_{\text {bin }}=64$ and $N_{\text {step }}=16$.

This treatment is essential for getting smooth time variation of amplitude and tune from the simulation as well as experiments. Fig. 2 (a) is a decay plot of Courant-Snyder invariant obtained from the tracking. The plot shows certain roughness which is not suitable in finding the decay constants. Fig. 2 (b) and (c) show tune and amplitude decay plots, respectively, they were treated by the explained method.

Amplitude decay plots in various conditions were fitted with the function $\alpha_{0} e^{-\left(\alpha_{1} n+\alpha_{2} n^{2}\right)}$, where $\alpha_{0}, \alpha_{1}$ and $\alpha_{2}$ are fitting variables and $\alpha_{2}$ gives tune-spread using (8) and (9). An example of fitting is shown in Fig. 2 (c).

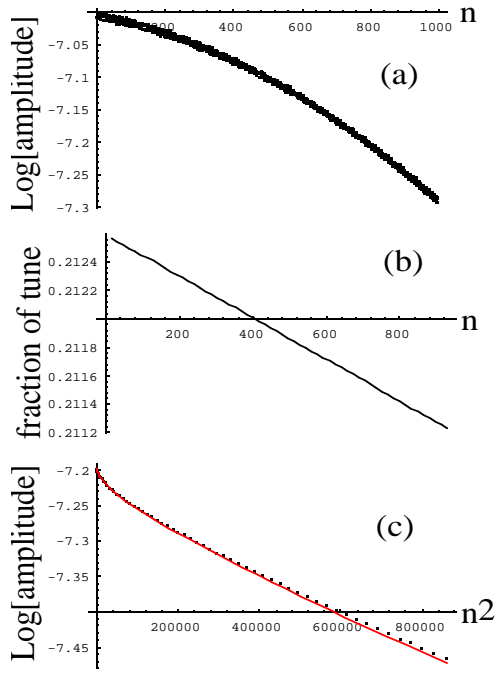

Figure 2: Example of the simulation. (a) decay plot of Courant-Snyder invariant. Position and angle information by the tracking and Twiss parameters are used. (b) decay plot of tune. (c) decay plot of oscillation amplitude and a fitted curve

\subsection{Optics for the simulation}

Nonlinear coefficients introduced by (1) were calculated using DA-package incorporated to SAD code [6] and listed in Table 1 for the three optics used in the simulation. 
Table 1: Nonlinear coefficients of optics for the simulation. Natural emittances and radiation damping rates are also given.

\begin{tabular}{|c|c|c|c|}
\hline & TRISTAN & $\begin{array}{c}\text { TRISTAN } \\
\text { Non- } \\
\text { interleaved }\end{array}$ & $\begin{array}{c}\text { KEKB-LER } \\
\text { Non- } \\
\text { interleaved }\end{array}$ \\
\hline$a_{x x}\left[\mathrm{~m}^{-1}\right]$ & 5356.91 & 52.28 & 5998.13 \\
$a_{x y}\left[\mathrm{~m}^{-1}\right]$ & -9299.00 & 44.493 & 13178.38 \\
$a_{x z}\left[\mathrm{~m}^{-1}\right]$ & 88.86 & 728.87 & 87.86 \\
$a_{y y}\left[\mathrm{~m}^{-1}\right]$ & 47106.96 & -59.76 & 29273.20 \\
$a_{y z}\left[\mathrm{~m}^{-1}\right]$ & 152.134 & 323.14 & -16.45 \\
$a_{z z}\left[\mathrm{~m}^{-1}\right]$ & 16.28 & 19.46 & 3.85 \\
\hline \hline$\varepsilon_{x}[\mathrm{~nm}]$ & 4.66 & 4.77 & 19.04 \\
$\varepsilon_{z}[\mathrm{~nm}]$ & 957.87 & 599.36 & 2431.12 \\
$\alpha_{x, y}$ & $0.91 \times 10^{-4}$ & $0.91 \times 10^{-4}$ & $1.29 \times 10^{-4}$ \\
\hline
\end{tabular}

Two of TRISTAN optics are almost same in linear optics, but nonlinear effect by sextupoles was canceled out very well in the non-interleaved one and nonlinear coefficients are much smaller than in the interleaved one.

In KEKB-LER optic where sextupoles are also noninterleaved, edge effect of the nearest quadrupoles to IP contributes to the nonlinear coefficient $a_{y y}$ largely. [5]

\section{RESULTS AND DISCUSSION}

\subsection{Simulation result the analytic treatment}

Results of the simulation are presented in Fig. 3 together with results of the analytic calculation by (6).

A good agreement between the simulation and the analytic treatment is found in Fig. 3 (a) for the interleaved TRISTAN optic. Also tune-spread depends on coherentamplitude linearly as expected by (4).

On the other hand, the non-interleaved optic of TRISTAN in Fig. 3 (b) gives completely different answer between the simulation and the analytic results. Tune-spread does not depend on amplitude linearly and suggests nonlinear terms higher than included in (1). The simulation could not explain the experimental results and confess that the simulation model is far from reality.

KEKB-LER optic shows a fairly good agreement between the simulation and the analytic results especially in case of small coherent-amplitude.

\subsection{Discussion}

The possibility of emittance measurement by nonlinear filamentation is demonstrated in optics where nonlinear coefficients of the lowest order are dominant. However when the lowest nonlinear coefficients are very small, nonlinear optics depend highly on errors and it becomes difficult to measure emittance by the proposed method.
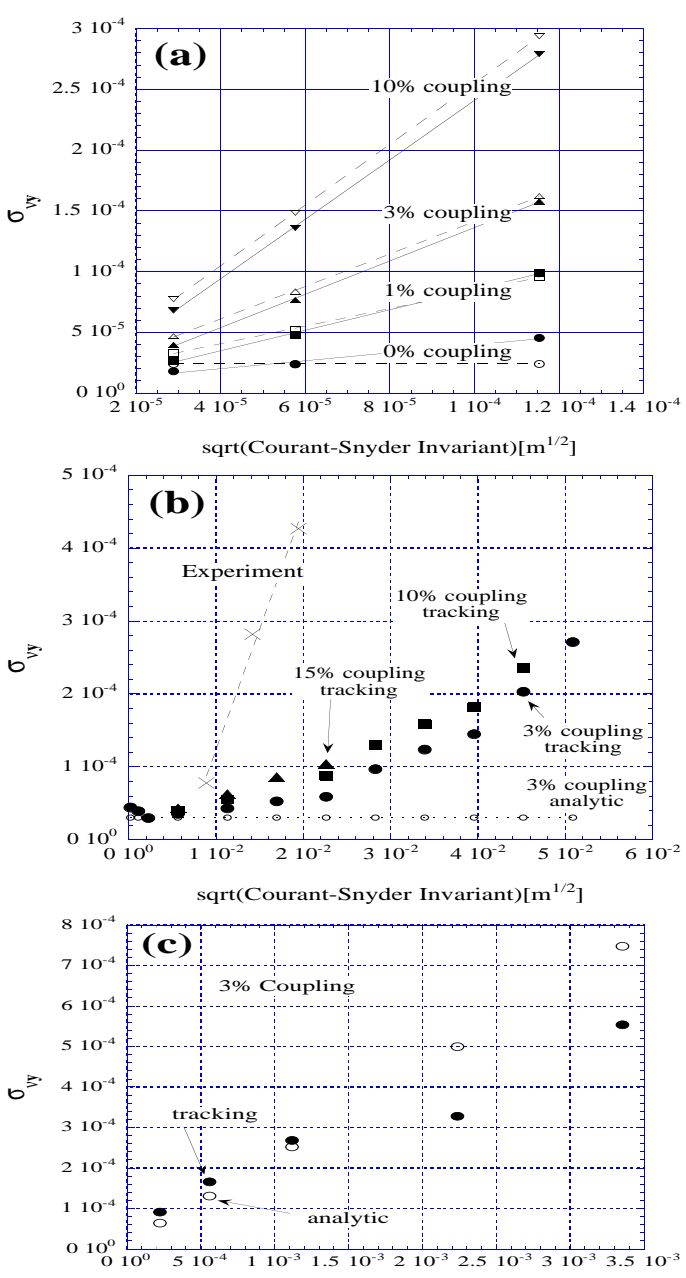

sqrt(Courant-Snyder Invariant) $\left[\mathrm{m}^{1 / 2}\right]$

Figure 3: Tune-spread vs. Coherent-amplitude. Be aware large difference of horizontal scale between interleaved and non-interleaved optics. (a) Interleaved optic of TRISTAN. The simulation and the analytic results are indicated solid and broken lines, respectively. By increasing coupling, tune-spread becomes larger as expected from (7). (b) Noninterleaved optic of TRISTAN. Experimental data are calculated from Fig. 1. (c) KEKB-LER optic.

\section{ACKNOWLEDGMENTS}

One of the authors (S. K.) enjoyed fruitful discussions with Prof. K. Yokoya on the distribution function.

\section{REFERENCES}

[1] M. Berz, SSC-166 (Part I), (1988).

[2] E. Forest, Particle Accelerators,24,91 (1989).

[3] S. Kamada, AIP Conference Proceedings 344, 1 (1995).

[4] N. Akasaka and S. Kamada, proc. of EPAC'96, 1141 (1996).

[5] K. Oide and H. Koiso, Phys. Rev. E47, 2010 (1993).

[6] K. Oide, H. Koiso and K. Ohmi, KEK Preprint 96-130 (1996).

[7] http://www-acc-theory.kek.jp/SAD/sad.html. 